\title{
Investigation of Coupled Heat Transfer and Electrothermal De-icing System of Helicopter Blade
}

\author{
Yang Bo \\ School of Aeronautic Science and Engineering \\ Beihang University \\ Beijing, China \\ yangbo505@ase.buaa.edu.cn
}

Fu Jianping

Equipment Development Office of Army Aviation

Department

Beijing, China

\author{
Chang Shinan \\ School of Aeronautic Science and Engineering \\ Beihang University \\ Beijing, China \\ snchang@buaa.edu.cn \\ Cheng Lin \\ School of Aeronautic Science and Engineering \\ Beihang University \\ Beijing, China \\ solitary.linna@gmail.com
}

\begin{abstract}
Numerical simulation of helicopter blade de-icing procession in typical conditions in two-dimensional was expanded. The Finite Volume Method was used to discretize the governing equations. The temperature distribution of the de-icing system was obtained by solving the coupled equations of external icing, internal heat conduction and the mixed ice-water phase transfer. The whole calculation process was based on Messinger model and the improved enthalpy method. Two observation points were placed at the stagnation point in the leading-edge area and out of the runback area on the lower surface to detect the ice thickness in these areas in order to evaluate the effect of the structure designed. The effects of different icing conditions on the temperature distribution of the de-icing system were analyzed. Further, taking into account of the deficiencies of the limited heat protected range and runback ice of the aimed simulation system, configuration development and numerical simulation on the heat protected range, the places of heating wires and the heat power distribution were presented.
\end{abstract}

Keywords-helicopter blade; numberical simulation;deicing; runback ice; surface temperature

\section{INTRODUCTION}

Helicopters are playing an increasingly important role both in military and civilian fields, for its flexible, mobile performance. As the helicopters' flight conditions (flight height, temperature and speed, etc.) in the icing envelope, flight accidents due to rotor blade icing occur frequently [1].

Helicopter rotor blades are smaller and thinner compared to fixed-wing normally; icing will cause more serious damage to the aerodynamic shape. Therefore, setting anti-icing and de-icing system is particularly important.

Electro-thermal de-icing is currently the most commonly used de-icing method for helicopter rotor blade [2]. As the electro-thermal de-icing system working, blade surface temperature rise up over the melting point of ice, so that the ice on the surface melts, with the formation of a thin liquid layer. Then ice will fall off from the frozen parts surface in the effect of the aerodynamic force and centrifugal force. However, when the electro-thermal deicing system works, as the range of thermal protection system or electric thermal power insufficient, it is easier to accrete runback ice behind the blade protection range, which will damage the aerodynamic performance of the airfoil, which will directly produce an effect on the normal hovering and flight safety.

Foreign investigators has made a lot of numerical calculation on electro-thermal de-icing, some have fundamental importance effect in the early study, as follows: Stallabrass[3]established the mathematical model of (flat) electric-thermal de-icing at the earliest, and study about the influence of the thickness ratio of inner insulating layer to the outer insulating layer on the electrothermal de-icing, get the optimal thickness ratio range; Masiulaniec[4]created a two-dimensional calculation model in 1987. The model using the method of conformal transformation, transferred the blade leading edge hierarchical de-icing structure to Cartesian coordinates, replaced the complex geometry by a rectangular structure, and overcome the calculation problem of curvature; Yaslik[5]established the three-dimensional simple electrothermal de-icing mathematical model in 1992. Investigate of electro-thermal de-icing is relatively less, among those investigates; Thomas Reid [6] et al. coupled external freezing with internal heat, calculated unsteady de-icing process, analyzed the influence of icing, overflow water and structure model on the temperature of de-icing surface.

Early years we were lack of recognition of the importance of aircraft icing protection. And the numerical calculation of the electro-thermal de-icing is very complicated. For these reason, study on the topic have started rather late in the domestic. Number of investigate relatively is less.

Given the complexity of the 3D air - water two-phase flow field generated by the rotor, there is no mature 
technology can completely simulate the 3D de-icing process investigate in home and abroad.

The angle of attack is biggest when the helicopter in hovering condition. Serious deterioration happens to the aerodynamic characteristics with the accretion of the runback ice, which will directly affect the flight safety and normal hovering. Therefore, investigate on the rationality of the design and work in the ice system in hover state is very important. In this paper, numerical calculation has been made for the electro-thermal de-icing of helicopter rotor blade in hover state. The calculation method of internal and external coupling is developed.

\section{MATHEMATICAL MODEL AND CALCULATION METHOD}

Electro-thermal de-icing system heating device is usually installed composite layer structure with the electric heater in the skin. Figurel shows the electro-thermal deicing heating pad structure model used in the paper which is composed with four parts, namely: the corrosion protection layer (wear-resisting layer), heating layer, insulating (internal and external insulation) layer and the support layer. The heating layer is seamless segmented into six heater strips. Heating position, number, is shown in figure 1. As the icing area of the front edge and the lower surface need pay great attention to, so two ice thickness monitoring point for detecting icing conditions, namely $\mathrm{A}$ and $\mathrm{B}$.

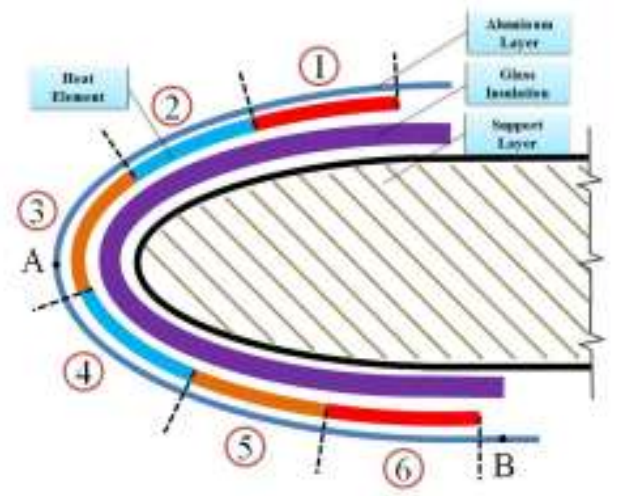

Figure 1. Structural representation of electro-thermal de-icing system

\section{A. The internal heat conduction simulation}

To heat conduction model make the following assumptions in this paper,

- The physical parameters of the each layer in the system materials unchanged;

- Ice (water) layer and between each solid layers keep good contact;

- After the ice layer with a certain thickness melted, the ice layer fell off.

The control thermal conductivity of enthalpy equation of the inner layers of the two-dimensional electro-thermal de-icing system is as follows:

$$
\frac{\partial H_{j}}{\partial t}=k\left(\frac{\partial^{2} t_{j}}{\partial x^{2}}+\frac{\partial^{2} t_{j}}{\partial y^{2}}\right)+q_{j}
$$

In this equation, $H_{j}$ refers to the total enthalpy value $(\mathrm{J}) ; t_{j}$ refers to the temperature of the control volume; $\mathrm{k}$ refers to the heat conductivity coefficient of the control volume $(W / m \cdot K)$; $\mathbf{j}$ said system layers, $q_{j}$ refers to the internal heat source $\left(\mathrm{W} / \mathrm{m}^{2}\right)$, except the heating layer, value of the inner heat source of other layers is 0 .

Enthalpy and temperature of solid layers of de-icing system distribute in the form of single value linear function, and temperature of ice (water) mixed layer in the process of phase transition is constant. The enthalpy and temperature is in nonlinear relationship, compared to the inner layers the heat transfer process is more complex. This paper uses the improved enthalpy method model analysis the heat transfer analysis of ice (water) mixing layer, assume that the temperature of the phase change process occurs in a small range, the ice (water) the relation of enthalpy and temperature are as follows:

Ice:

$$
\mathrm{H}_{i}=\rho_{i} \cdot c_{p i} \cdot t \quad\left(t<t_{m}\right)
$$

Water:

$$
\begin{gathered}
\mathrm{H}_{i m}=\rho_{i} \cdot c_{p i} \cdot t_{m} \\
\mathrm{H}_{w m}=\rho_{w} \cdot\left(c_{p i} \cdot t_{m}+L\right) \\
\mathrm{H}_{w}=\rho_{w} \cdot c_{w} \cdot\left(t-t_{m}\right)+\rho_{w} \cdot\left(c_{p i} \cdot t_{m}+L\right) \quad\left(t>t_{m}+\Delta t\right)
\end{gathered}
$$

Ice water mixed layer:

$$
\mathrm{H}_{m}=\mathrm{H}_{i m}+\left(t-t_{m}\right)\left(\mathrm{H}_{w m}-\mathrm{H}_{i m}\right) / \Delta t \quad\left(t_{m} \leq t \leq t_{m}+\Delta t\right)
$$

Among them, the subscript i, m, w are on behalf of ice, mixture of ice and water, water respectively, $\rho$ for density $\left(\mathrm{kg} / \mathrm{m}^{3}\right)$, c for the specific heat at constant pressure $(\mathrm{J} / \mathrm{kg} \cdot \mathrm{K})$, L for the phase change latent heat of ice $(\mathrm{J} / \mathrm{kg}), t_{m}$ for the phase transition temperature of water $(\mathrm{K})$, $\Delta t$ for the phase transition temperature range.

Boundary conditions of thermal control equation (1) as follows:

Set the external heating surface to the third kind boundary:

$$
-k \frac{\partial t}{\partial y}=h_{e f f}\left(t_{s}-t_{\infty}\right)
$$

Set the internal heating surface to the adiabatic boundary:

$$
\frac{\partial t}{\partial x}=0
$$

Heating boundaries at both ends:

$$
\frac{\partial t}{\partial x}=0
$$

Among them, $t_{\infty}$ represent environment temperature (K).

Thermal simulation using TDMA method and ADI Block correction technique, that the discrete equations of heat conduction was calculated, specific solving methods as shown in literature [7].

\section{B. The external icing simulation}

Given some time in the process of de-icing with icing phenomenon, therefore, it needs to simulate the icing progress on skin surface. Icing calculation is mainly separate d into the flow field calculation, water droplets collision characteristics and heat transfer coupling calculation.

Fluid flow required to comply with the mass, momentum and energy conservation equation, for clean airfoil profile and the calculation of flow field after icing, 
this paper selects the $\mathrm{S}$ - A equation turbulent model and using FLUENT, the control volume method is adopted to discrete, and the discrete equation is solved using the SIMPLE method. In this paper the ICEM is used to partition calculation domain, in which the far field there was divided into 15 times before and after the chord $\mathrm{C}$ grid structure, solid part for multi-layer BLOCK structure grid, after the grid independence analysis, determine the amount of far field and solid computing domain grid. Calculation of airfoil profile is OA213 airfoil [8], whose chord length is $0.5334 \mathrm{~m}$. Set the air-water inlet boundary condition to pressure far field, set airfoil wall to no slip wall, set the upper and lower boundary of the computational domain to the adiabatic condition, as shown in Fig. 2.

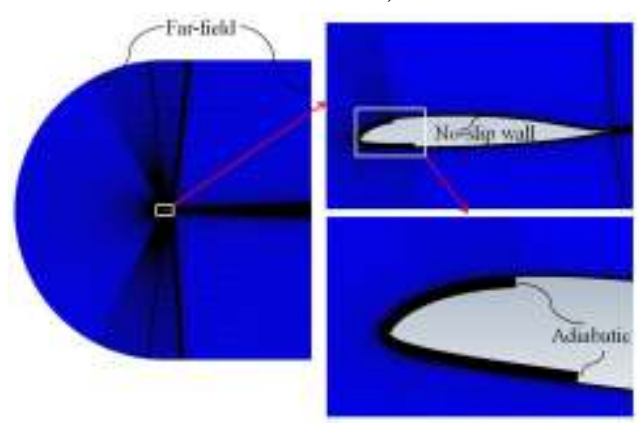

Figure2. Far field and solid calculate domain and its boundary conditions

In two-dimensional water-drop flow field, by reason of no need to do air field coupling calculation, using the Lagrangian method will save the computing resources, compared to the Euler method. Aimed at the ice problem, established frozen water droplets movement equation as followed:

$$
\begin{aligned}
& \frac{d^{2} \vec{x}_{d}}{d t^{2}}+\frac{C_{\mathrm{d}} R \mathrm{e}_{\mathrm{w}}}{24} \frac{18 \mu}{d^{2} \rho_{\mathrm{w}}} \frac{d \vec{x}_{d}}{d t} \\
& =\frac{\rho_{\mathrm{w}}-\rho_{\mathrm{a}}}{\rho_{\mathrm{w}}} \vec{g}+\frac{C_{\mathrm{d}} R \mathrm{e}_{\mathrm{w}}}{24} \frac{18 \mu}{d^{2} \rho_{\mathrm{w}}} \vec{u}
\end{aligned}
$$

The initial conditions:

$$
\begin{aligned}
\vec{x}_{d}(0) & =\left(x_{0}, y_{0}\right) \\
\left.\frac{d \vec{x}_{d}}{d t}\right|_{t=0} & =\vec{V}_{\infty}
\end{aligned}
$$

Among them, $C_{d}$ refers to the drag coefficient, $R e_{w}$ refers to the Reynolds number of droplets relative to air, $\mu$ refers to the air viscosity $(\mathrm{Pa} \cdot s)$. Based on classic Messinger ice model, the thermal mass balance of the freezing process on the external surface was analyzed, specific solving methods as shown in literature [9][10].

\section{Internal and external coupling calculation method}

Under icing conditions, the heat transfer process on the external surface of blade is complex, so in this paper we introduced the concept of equivalent heat transfer coefficient $_{h_{\text {eff }}}$; describe the skin external complex heat transfer process in the form of a formula (13). Thereby finish the external coupling the internal solid heat conduction to the complex external heat transfer, and calculate the temperature field of the skin surface.

$$
Q=h_{e f f} \Delta s\left(t_{s}-t_{\infty}\right)
$$

According to the above formula, we can obtain $h_{\text {eff }}$; in the formula, $Q=\sum Q_{i}\left(t_{s}\right)$ is the sum of all the outside heat flow; $t_{s}$ refers to the temperature of the external surface of skin $(\mathrm{K}), t_{\infty}$ refers to the far field environment temperature (K).

Coupling iteration proceeds according to the following steps:

As shown in Fig.3, firstly, assume the initial skin surface temperature to be $t_{s}{ }^{0}$, obtain the temperature $t_{s}^{1}$ on external surface of the skin of the airfoil profile model, by external freezing module iterative calculation and the equivalent heat transfer coefficient $h_{\text {eff }}{ }^{0}$ and the thickness on the skin surface $h^{0}$. Then call the internal thermal module, set $t_{s}{ }^{1}$ and $h_{\text {eff }}{ }^{0}$ as the external boundary conditions of the control-volume with solid heat conduction calculation. Do iterative calculation after the conformal transformation of the $2 \mathrm{D}$ solid, we obtain the new surface temperature $t_{s}^{2}$. to reassign the value of $t_{s}^{2}$ unit control volume of the external icing calculation module, through the balance of heat and mass transfer and after ice iterative calculation, update the result and obtain $t_{s}{ }^{3}, h_{e f f}{ }^{1}, h^{1}$, now based on the convergence criterion $\left|t_{s}^{k+1}-t_{s}^{k}\right|<\sigma$ ( $\sigma$ is a given constant $)$, judge whether the surface temperature convergent. If not, repeat the above process until the surface temperature convergent. After the calculation, we obtain the current temperature of the surface. If you would like to get the distribution of the surface temperature all the moments, then repeat the above process in the next computational time step.

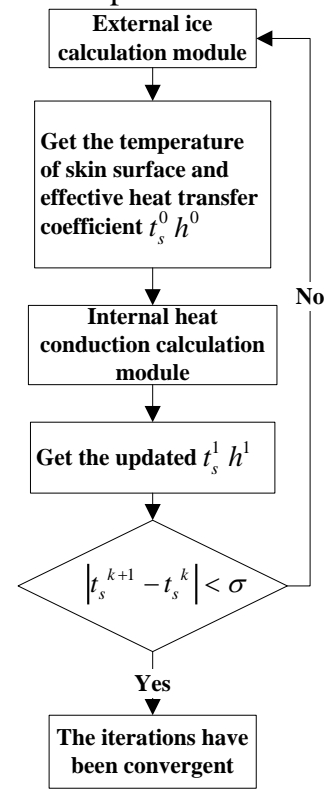

Figure3. Iterative calculation diagram

\section{THE EXAMPLE RESULTS AND ANALYSIS}

This article chooses the experimental results of paper [11] to verify the correctness of the simulation results in this paper. As shown in Figure 4, the change trend of temperature with time is calculated with experimental results given in literatures are basically the same, the 
extreme value of temperature slightly higher than the experimental results, the losing time of ice layer of is also very consistent. So the calculation results are credible.

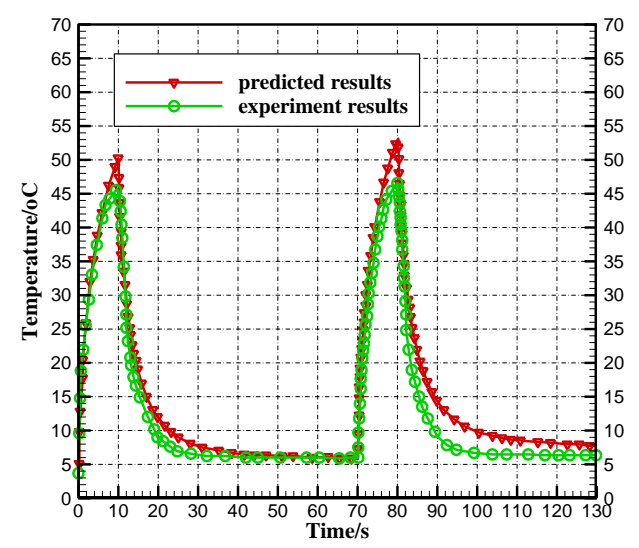

Figure4. Results of this paper compared with the literature

This article selects a certain cross of helicopter blade section as the investigate object, analyzes the influence of parts of the freezing parameters (temperature, pressure, water droplets diameter, liquid water content, etc.) on the de-icing system, and select to investigate some typical state, as shown in Table 1. As Helicopter rotor blade rotates, in different position of the circumferential angle, the angle of attack of the selected section is different, for the convenience of calculation; this article selects the mean value of each circumferential position corresponding angle of attack as the angle of airfoil. Each heater strip heats in turn, heating in the order (3) - (2) - (4) - (1) - (5)-(6), the heating time is the same as the same calculation condition. Heating time set mainly depends on the environment temperature, environment temperature is lower, the longer the heating time. When one heater strip is heating, others do not work.

\begin{tabular}{|c|c|c|} 
Table1 calculation conditions \\
\begin{tabular}{|c|c|c|}
\hline NO. & Case 3 & Case8 \\
\hline Temperature $(\mathrm{K})$ & 263.15 & 253.15 \\
\hline $\begin{array}{c}\text { LWC(liquid water } \\
\text { content })(\mathrm{g} / \mathrm{m} 3)\end{array}$ & 0.43 & 0.1 \\
\hline $\begin{array}{c}\text { Water droplet } \\
\text { diameter }(\mu \mathrm{m})\end{array}$ & 20 & 40 \\
\hline $\begin{array}{c}\text { Heating } \\
\text { time(seconds })\end{array}$ & 18 & 30 \\
\hline
\end{tabular}
\end{tabular}

Both cases have same pressure, angle of attack, velocity and heating power, their values are 47217(pa), $4.5\left(^{\circ}\right), 60(\mathrm{~m} / \mathrm{s})$ and $30(\mathrm{kw} / \mathrm{m} 2)$ respectively.

\section{A. Local droplet collecting coefficient}

Local droplet collecting coefficient reflects the water collecting ability of each point infinitesimal on the surface, has a great influence on calculating icing and design of protection range. In this paper, results are shown in Figure 3 . As we can see from case 1 , case 2 , as the droplets diameter increased from $20 \mu \mathrm{m}$ to, $40 \mu \mathrm{m}$, water droplets collision limit continually widening, and local collection coefficient increased. When the diameter reaches to $40 \mu \mathrm{m}$, the collision position of water droplets has beyond the corresponding heat boundary (as shown in abscissa $0.13 \mathrm{~m})$.

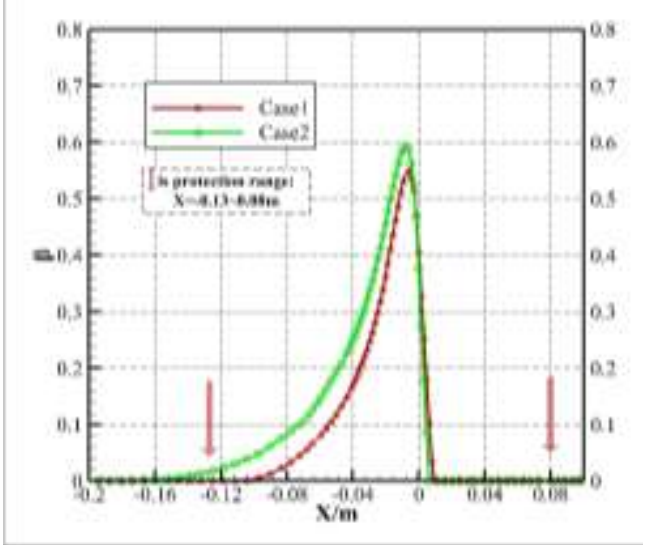

Figure5. Results of local water droplets collection coefficient

\section{B. Ice accretion thickness}

When ice thickness on skin surface is too serious, it will damage the aerodynamic shape of helicopter blade, and affects the aerodynamic characteristics, such as lift-todrag ratio, and equilibrium torque.

Fig. 6 shows that, the contrast of the ice thickness between both states, when heater strip (6) finished heating, which means a heating period just ended. The reason why choosing this moment as the typical moment to analysis is that at this time the stagnation point have been cooling for the longest time, and the water collecting ability near the stagnation point is the strongest, therefore, the icing condition is the most serious.

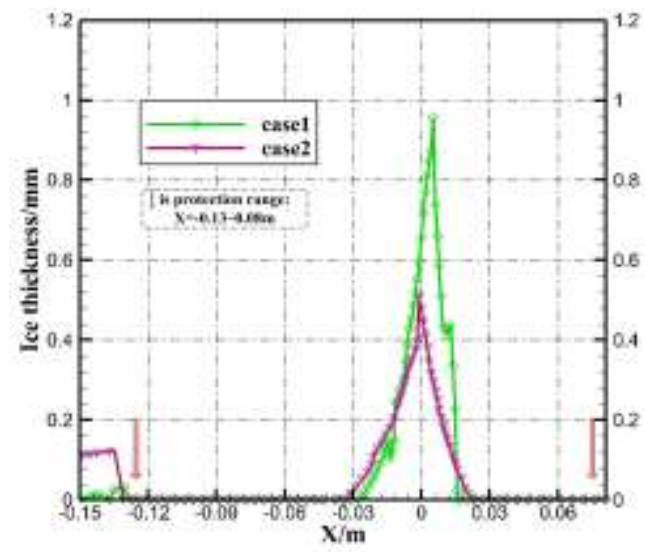

Figure6. The ice thickness after one period

In order to verify the system is working reasonably and effectively, in this article in the process of calculation has set up A, B two monitoring points, used to monitor the ice thickness in dangerous area. Among them, the point A located on the leading edge near the aerodynamic stagnation point, and Point B located out of the protection area of the lower surface near the border (see fig1.).

Fig7. shows the situation of the ice thickness at A and $B$ points change as time go on of case 1 . Ice thickness at Point A presents cyclical change over time. In each heating cycle, when the heater strip (3) heating ends, the ice on the surface of the skin can be completely eliminated. As the existence of afterheat after the power off, cooperating the reasonable heating control law, the largest ice thickness slightly decrease from the first cycle to the second cycle, 
then steady at $0.54 \mathrm{~mm}$, which indicates that system design in A point where the most serious icing place effective.

The ice thickness at Point B increased over time on the whole time. However, in the process of de-icing system work, due to the heater strip (6) near the point $\mathrm{B}$ heating cyclically, and the thermal conductivity of the skin, the ice thickness at point B presents cyclicality decreased. But as the time goes on, the ice thickness shows a trend of growth, we can predict that as the icing time gets longer, the ice thickness at point $\mathrm{B}$ will continue to increase, endanger the flight safety. The defect of protection system design, leading to the runback ice at point B.

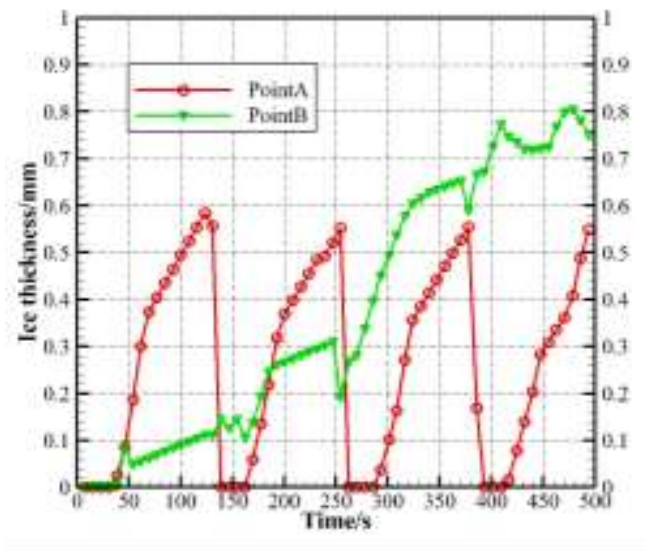

Figure7. Ice thickness of case1 icing monitoring points

\section{Surface temperature}

When de-icing system works normal, the ice layer closing to the heating area of skin will melt and fall off, the surface temperature is the main judgment of measure this process, so it need to analyze the surface temperature to evaluate whether the work of the system is valid. What is more, when the surface temperature is too high, it will over use the precious energy on aircraft. In order to determine whether the heating power needs to be further improved, it also needs to analyze the surface temperature of the skin. Fig. 8 shows calculation results of skin surface temperature that the heating strip (3) just finished heating.

The velocity, angle of attack and pressure of cases 1,2 are the same, and the liquid water content decreases in turn, and the average droplet diameter increases in turn. Two peak values appeared at the position of heater strip (6), (3) on the external skin surface temperature distribution corresponding to the heating areas, which is difference compared to the rest of the positions obvious. Beyond the collecting range of water, the surface temperature is mainly affected by heat convection of air, and the water droplet collection $(\beta)$ is minuteness on the upper surface.

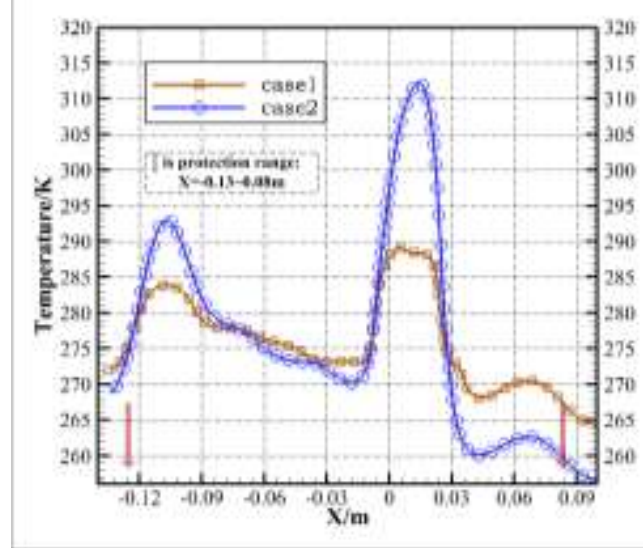

Figure8. Surface temperature distribution

\section{CONCLUSION}

In this article, we investigated the helicopter rotor blade electro-thermal de-icing problems, using the equivalent heat transfer coefficient $h_{\text {eff }}$ and the coupling method between internal and external flows. The skin surface temperatures were calculated, the influence of different icing conditions on the ice thickness and surface temperature researched.

Main conclusions are as follows:

- When the thermal protection of system is insufficient, runback ice will occur. The simulation shows that the ice accretion thickness is approximately linear increase trend as the time go on.

- The computed results show that the coupling method, developed in this article, between internal and external calculation of skin surface temperature is effective. Skin surface temperature was synthetically influenced by parameters such as the environmental temperature, the diameter of water droplets, and the liquid water content, and they all exerting important influence on results.

- Reasonable electro-thermal de-icing system design has a significant effect on the system energy consumption and performance. If the energy of protection system is not enough, runback ice will be frozen on the wing and cause serious consequences.

\section{REFERENCES}

[1] Green, S.D.A study of U. S. inflight icing accidents and incidents.[R] 1048-1073

[2] Qiu Xiegang, Han Fenghua. Aircraft anti-icing system [M]. Beijing. Aviation professional teaching material review group, 1985

[3] Stallabrass J R. Thermal aspects of de-icer design [C]. The international helicopter icing conference, ottawa, Canada, May 23, 1972

[4] Masiulaniec K C. A numerical simulation of the full twodimensional electrothermal de-icer pad[D]. Toledo: University of Toledo, 1987

[5] Yaslik A D, DeWitt K J, Keith T G. Further developments in threedimensional numerical simulation of electrothermal de-icing systems [R].AIAA-92-0528, 1992

[6] Reid, T., G.S. Baruzzi, and W.G. Habashi, FENSAP-ICE: unsteady conjugate heat transfer simulation of electrothermal de-Icing[J]. JOURNAL OF AIRCRAFT. 49(4): 1101-1109.2012 
[7] Chang Shinan, Ai Suxiao, Huo Xiheng, Yuan Xiugan. Improved simulation of electrothermal de-icing system [J]. Journal of Aerospace Power.2008.23(10):1753-06

[8] UIUC Airfoil coordinates Database. [DB/OL]. http://aerospace.illinois.edu/m-selig/ads/coord_database.html.

[9] X. Chi, Y. Li, H. Chen. A comparative study using CFD to predict iced airfoil aerodynamics[R]. AIAA 2005-1371.2005

[10] Yang Qiuming. Numerical Simulation of Airfoil Icing and Analysis Its Effects on Aerodynamic Performance[D].Beijing: Beijing University of Aeronautics and Astronautics,2009.

[11] Leffel K.L. A Numerical and Experimental Investigation of Electrothermal Aircraft De-icing [D]. M.Sc. Thesis, University of Toledo, Toledo, Ohio, Nov. 1985 